REVIEW

\title{
Stress echocardiography for the diagnosis and risk stratification of patients with suspected or known coronary artery disease: a critical appraisal. Supported by the British Society of Echocardiography
}

\author{
R Senior, M Monaghan, H Becher, J Mayet, P Nihoyannopoulos
}

Heart 2005;91:427-436. doi: 10.1136/hrt.2004.044396

Stress echocardiography today has matured into a robust and reliable technique not only for the diagnosis of suspected coronary artery disease (CAD) but also for the accurate risk stratification of patients with suspected and established CAD. This is mainly because of rapid advances in image acquisition, digital display, and the development of harmonic and contrast imaging. Stress echocardiography today is also utilised in patients with heart failure both for assessing the cause of heart failure and determining the extent of hibernating myocardium. With advances in myocardial perfusion imaging, stress echocardiography now allows simultaneous assessment of myocardial function and perfusion. Tissue Doppler imaging allows quantitation of wall motion. Ready availability and reliability makes stress echocardiography a cost effective technique for the assessment of CAD.

See end of article for authors' affiliations

Correspondence to: Dr Roxy Senior Department of Cardiovascular Medicine, Northwick Park Hospital, Harrow HAl 3UJ UK, UK; roxy.senior@virgin.net

Accepted 11 October 2004
$E$ xercise ECG is the most widely used technique for the diagnosis and risk stratification of patients with suspected or known coronary artery disease (CAD). However, it is well recognised that only $40 \%$ of patients can perform a truly diagnostic exercise test and that the occurrence of transient ST segment depression, which is regarded as the hallmark of myocardial ischaemia, is affected by factors other than myocardial ischaemia. ${ }^{12}$ These factors, and the fact that ST-T changes occur late in the ischaemic cascade, makes exercise ECG a test of modest diagnostic accuracy for the detection of CAD. It is even less accurate in patients who have an intermediate likelihood of CAD, which comprises the bulk of the population that requires accurate risk stratification. ${ }^{3}$

Stress echocardiography was introduced in the early 1980s and has matured over the years as a reliable and cost effective method for both the diagnosis and risk stratification of patients with suspected or known CAD. As a result, the uptake of stress echocardiography as the non-invasive method of choice for the assessment of CAD has increased exponentially worldwide and is continuing to expand. In the USA alone approximately three million stress echocardiographs were performed last year. The hallmark of myocardial ischaemia during stress echocardiography is the occurrence of reduced systolic wall thickening when myocardial oxygen demand exceeds myocardial blood supply. This precedes the occurrence of chest pain and ST-T changes. The induction of reduced regional systolic wall thickening is specific for CAD. The advent of harmonic imaging, tissue Doppler techniques, and the availability of contrast microbubbles together with the advancement of digital imaging/display technology, have made stress echocardiography today a reliable and reproducible technique for the assessment of CAD. ${ }^{4-6}$

\section{Stress protocol and interpretation}

Stress echocardiography may be performed in conjunction with dynamic exercise (treadmill or bicycle). In patients who are unable to exercise, pharmacological agents may be used, such as dobutamine or dipyridamole. With any form of stress testing echocardiographic images are first acquired digitally during rest in parasternal and apical views. These views of the heart visualise all three vascular territories. Subsequently, stress images are acquired during low, intermediate, and peak stress, except for treadmill exercise where images may be acquired immediately (6090 seconds) after peak stress for optimal imaging. Stress and rest images are arranged digitally side by side in each view for analysis and archiving.

Rest and stress images are interpreted for global and regional left ventricular (LV) size, shape, and function. A normal response is when, during stress, the LV size becomes smaller compared to rest, while the shape is maintained and there is increased endocardial excursion and systolic wall thickening (fig 1). Figure 2 shows an example of a patient who had undergone exercise echocardiography demonstrating a dilated LV cavity with change in shape, and notable reduction of systolic wall thickening of the septum, anterior, and inferior wall, suggestive of multi-vessel disease. Prolonged persistence of systolic wall thickening abnormality may also identify severe CAD. ${ }^{7}$

Dobutamine, which is a sympathomimetic agent, is particularly useful in patients with an existing resting wall thickening abnormality. Low doses of dobutamine increases myocardial

Abbreviations: CAD, coronary artery disease; LV, left ventricular; SPECT, single photon emission computed tomography 

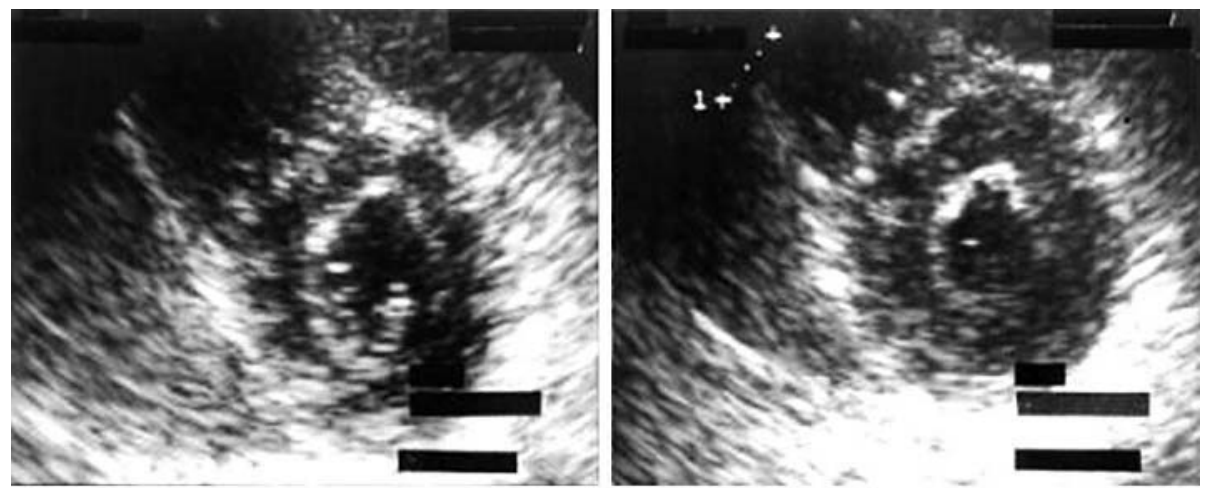

Figure 1 Normal left ventricular (LV) response during dobutamine stress in the short axis view. Upper left (rest), upper right (low dose of dobutamine), lower left (high dose of dobutamine) and lower right post-stress.
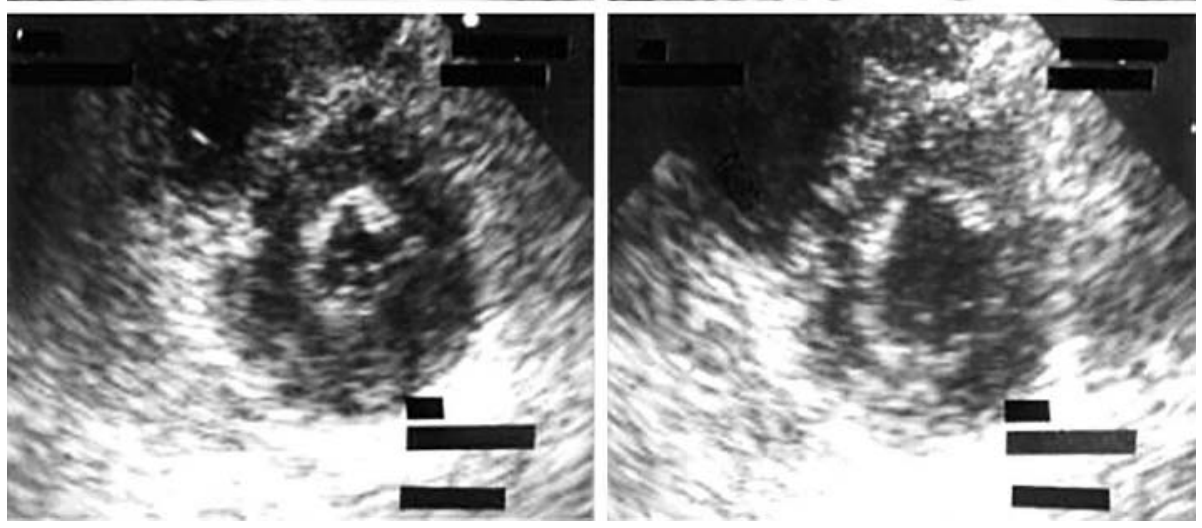

perfusion, recruits potentially contractile myocardium, and hence increases myocardial contractility in dysfunctional myocardium if there is sufficient contractile reserve (viability). At high dose, however, dobutamine increases myocardial oxygen demand and in the presence of flow limiting stenosis will result in demand/supply mismatch leading to myocardial ischaemia, resulting in deterioration of regional function (biphasic response). ${ }^{8}$ Thus, dobutamine at low doses depicts the presence of myocardial viability, while at high doses uncovers myocardial ischaemia (fig 3). Dobutamine is also widely used as an alternative test in patients who are unable to exercise. Figure 4 describes the various myocardial responses to dobutamine in patients with and without resting wall thickening abnormality. Dipyridamole may be used as an alternative to dobutamine but it produces infrequent wall thickening abnormalities even in the presence of significant flow limiting coronary stenosis. ${ }^{9}$

\section{Diagnosis of CAD}

The coronary arteriographic cut off of luminal diameter stenosis at which wall thickening abnormalities occur is $54 \%$ for exercise, $58 \%$ for dobutamine, and $60 \%$ for dipyridamole. ${ }^{10}$ The sensitivities for the detection of CAD (cut off of $>50 \%$ luminal diameter stenosis) were $85 \%, 80 \%$, and $78 \%$ with specificities of $77 \%, 86 \%$, and $91 \%$, for exercise, dobutamine, and dipyridamole stress results. ${ }^{10}{ }^{11}$ However, it is clear that the diagnostic accuracy of any test varies according to the pre-test likelihood of CAD in the population tested. For example, if a pre-test likelihood of CAD is low then a positive test is likely to be false and conversely if the pre-test likelihood is high a negative test is likely to be a false negative. It is also clear that non-invasive tests will have the greatest clinical value only in the population with intermediate likelihood of CAD. Radionuclide single photon emission computed tomography (SPECT), which is an alternative non-invasive technique, is also widely used to assess CAD. However, its widespread use is limited by cost, radiation, and relative lack of availability. In a meta-analysis based on 44 studies where the two techniques were directly compared in patients without prior acute myocardial infarction and without past history of known ischaemic heart disease-that is, in patients with high to intermediate
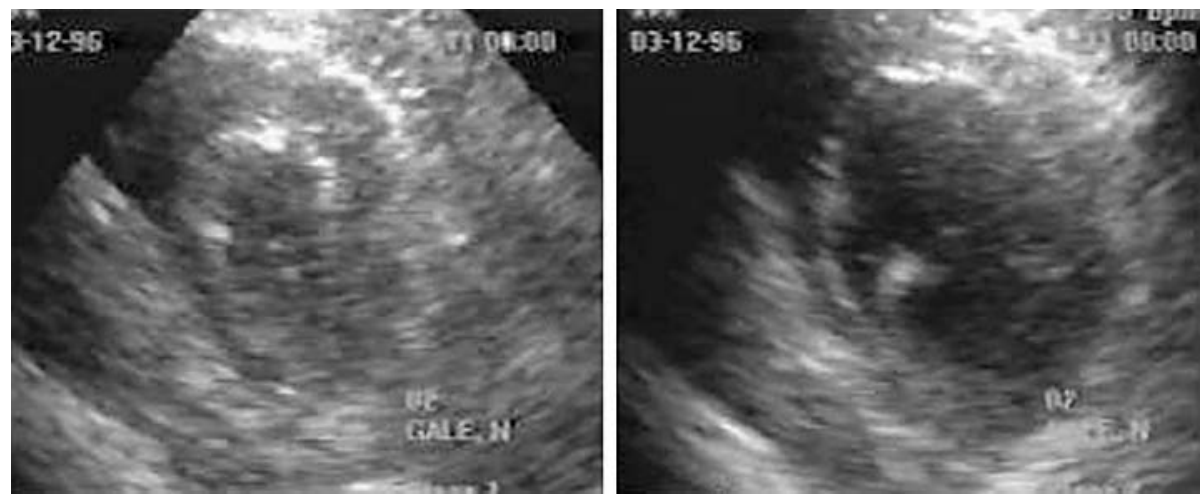

Figure 2 Abnormal LV responses during exercise. Left, at rest; right, 60 seconds after exercise. LV is dilated with severe systolic wall thickening abnormalities affecting septum, anterior, and inferior wall, suggestive of multi-vessel disease. 

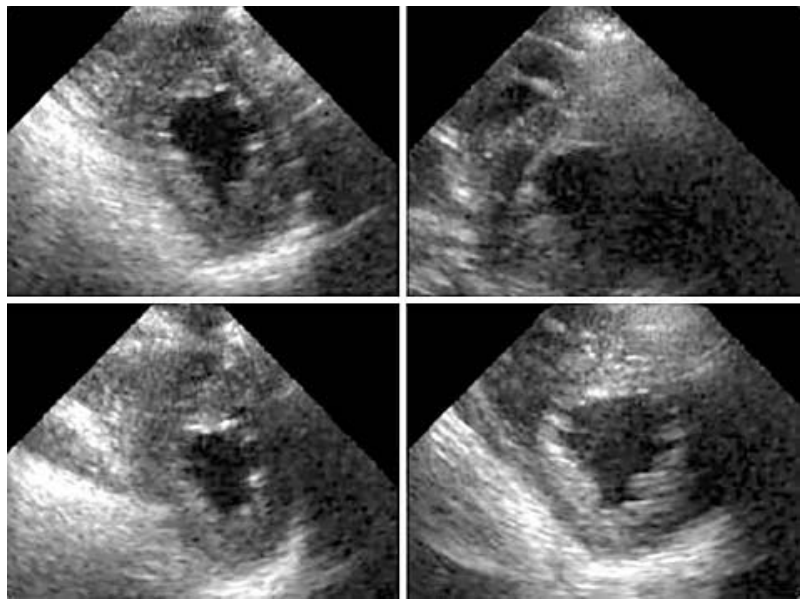

Figure 3 Biphasic response during dobutamine stress upper left (rest), upper right and lower left (low doses of dobutamine), and lower right (high doses of dobutamine). There was improvement of systolic wall thickening in a patient with LV dysfunction with deterioration of both anterior and inferior wall thickening during high dose.

likelihood of CAD—stress echocardiography had a sensitivity of $85 \%$ (95\% confidence interval (CI) $83 \%$ to $87 \%$ ) with a specificity of $77 \%$ ( $95 \%$ CI $74 \%$ to $50 \%$ ), while SPECT had a sensitivity of $87 \%$ (95\% CI $86 \%$ to $88 \%$ ) with a specificity of $64 \%(95 \%$ CI $60 \%$ to $68 \%) .{ }^{12}$ In models comparing the discriminatory abilities of stress echocardiography and SPECT versus exercise testing with ECG alone, both stress imaging techniques performed significantly better than exercise testing. However, the incremental improvement in performance was greater for stress echocardiography $(3.43 \%$, $95 \%$ CI $2.74 \%$ to $4.11 \%$ ) than for SPECT $(1.49 \%$, $95 \%$ CI $0.91 \%$ to $2.08 \%)$. $^{12}$

SPECT detects a relative reduction in myocardial blood volume that occurs earlier than wall thickening abnormality in the ischaemic cascade. Despite this, SPECT has not been shown to be significantly superior to stress echocardiography in terms of sensitivity and detecting the extent of CAD. There was no significant difference between the two techniques in the sensitivity for location of CAD $(72 \% v 69 \%)$ and in the prediction of multi-vessel CAD (58\% v 50\%). ${ }^{13}$ The lack of significant difference in sensitivity between SPECT and echocardiography may be explained by two major factors: SPECT requires a difference of at least $35 \%$ in myocardial blood volume before a relative perfusion defect is appreciated and spatial resolution is poor (about $12 \mathrm{~mm}$ ). Conversely, echocardiography has far greater spatial resolution (about $2 \mathrm{~mm}$ ) and wall thickening abnormalities are assessed independently in each vascular territory, unlike SPECT. Stress echocardiography has significantly superior specificity

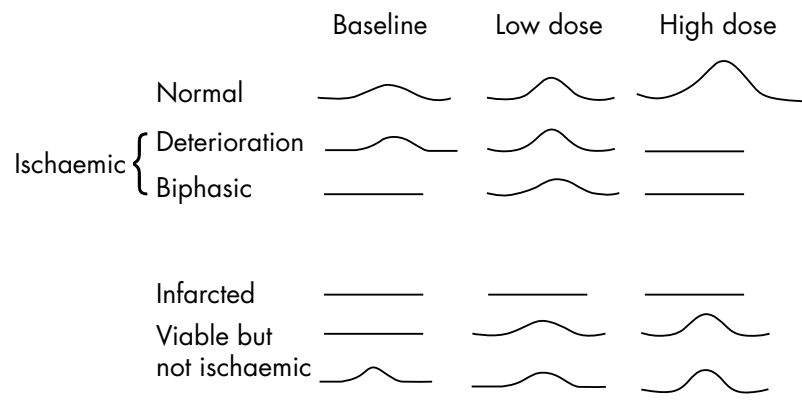

Figure 4 Different myocardial responses to dobutamine. compared to SPECT for detection of CAD in all patient groups.

Another attribute of an ideal non-invasive test is to identify patients at high risk of having multi-vessel CAD because these patients have poor outcome even in the presence of normal LV function. Clinical variables on their own can identify such patients and exercise ECG also improves the detection of multi-vessel disease. Does stress echocardiography improve the detection of multi-vessel disease over and above the clinical and exercise data? In a study by Rogers and colleagues, ${ }^{14}$ the presence of wall motion abnormality in multiple vascular territories during exercise echocardiography identified additional patients with multi-vessel disease. This was also shown by another study using dobutamine echocardiography. ${ }^{15}$

\section{Hypertension and sex}

Stress ECG has an unacceptably high incidence of false positive result in patients with hypertension and also in women. ${ }^{1}$ A recent large study assessed stress echocardiography for the detection of CAD in patients with LV hypertrophy. In this group the majority were hypertensive. The overall sensitivity and specificity was $85 \%$ and $87 \%$, respectively. ${ }^{16}$ However, in patients who demonstrated notable reduction in cavity diameter caused by concentric remodelling at rest, stress echocardiography had a low sensitivity. ${ }^{16}$ In a comparative pooled analysis, the sensitivity of echocardiography was $74 \%$ compared to $87 \%$ with SPECT, however the specificity of echocardiography was superior to SPECT (85\% $v$ $44 \%) .{ }^{13}$ In women, stress echocardiography showed a trend towards better sensitivity $(80 \%)$ and specificity (89\%) compared to SPECT $(71 \%$ and $72 \%){ }^{13}$ The accuracy of SPECT appears to be decreased in women because of breast tissue attenuation artefact and smaller LV chamber size.

\section{Risk stratification}

\section{Outcome of normal stress echocardiography}

The outcome of normal stress echocardiography has been assessed in several large studies. In a study of 2829 patients with normal rest and exercise echocardiography followed up for six years, mortality was less than $1 \%$ per annum. ${ }^{17}$ In another study of 1325 patients overall survival of patients after normal exercise echocardiography was significantly better than that of an age and sex matched group obtained from life tables $(p<0.0001) .{ }^{18}$ The cardiac death and nonfatal acute myocardial infarction rate per year of follow up was $0.5 \%$. The cardiac mortality in 4479 patients with normal pharmacological stress echocardiography was $0.7 \%$ per year in another study. ${ }^{19}$ In a smaller study involving 252 patients studied in a busy district general hospital in UK, the patients with a normal stress echocardiogram, in a group with a predominantly intermediate pre-test likelihood of CAD, who were followed up for a mean of 2.8 years, cardiac mortality was only $0.4 \%$ per year and a combined end point of cardiac mortality and acute myocardial infarction was $0.8 \%$ per year. ${ }^{20}$ In this study, exercise ECG was positive in $32 \%$ of patients.

In two head to head studies comparing stress echocardiography and stress myocardial perfusion imaging, the outcomes of normal scans were identical. ${ }^{21} 22$ Thus, in patients with suspected CAD, a normal stress echocardiogram confers an excellent prognosis. Approximately $10-30 \%$ of patients are reported to have normal coronary arteries on coronary angiography. Coronary arteriography may be safely avoided in all patients if stress echocardiography is normal. Stress echocardiography may therefore function as a gatekeeper for referral to invasive strategies because it has the ability to identify low risk patients who have an excellent prognosis when treated conservatively. In this context, stress 


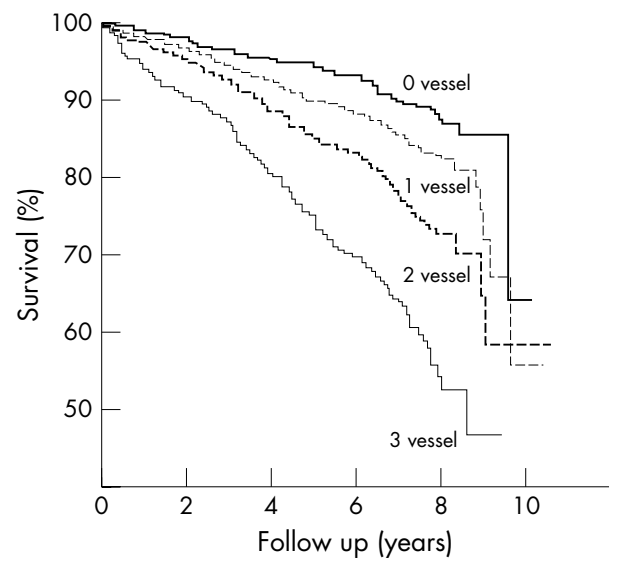

Figure 5 Mortality of patients according to the total extent of wall motion abnormalities (summed stress score) at peak stress ( $\mathrm{n}=5375$ patients). ${ }^{17}$

echocardiography may be regarded as a highly cost effective tool. This aspect will be discussed further in a subsequent section of this review.

\section{Outcome after abnormal stress echocardiography}

Stress echocardiography can quantify the severity and extent of myocardium in jeopardy and thereby predict risk. ${ }^{17} 182324$ The total amount of myocardium in jeopardy can be assessed at peak stress by adding the peak stress score and dividing by the number of segments (summed stress score) assessed in a polar map using the American Society of Echocardiography 16 segment LV model. In a study involving 5375 patients undergoing exercise echocardiography, in which the patients were followed up for 10.6 years, the extent of wall motion abnormality, calculated as summed stress score, incrementally predicted cardiac mortality (fig 5). ${ }^{17}$

In clinical practice, the important question is whether any additional test provides incremental and independent information over and above clinical data in patients with suspected or even known CAD. Exercise ECG has been shown to provide additional information. The Duke treadmill score which uses exercise time, ST-T segment changes, and anginal symptoms, is a well established scoring system for identifying risk after a treadmill exercise test using the Bruce protocol. A high Duke score confers good prognosis while a low score identifies patients at high risk. In a study by Marwick et al, information derived from the exercise echocardiography was incremental and independent of clinical data,

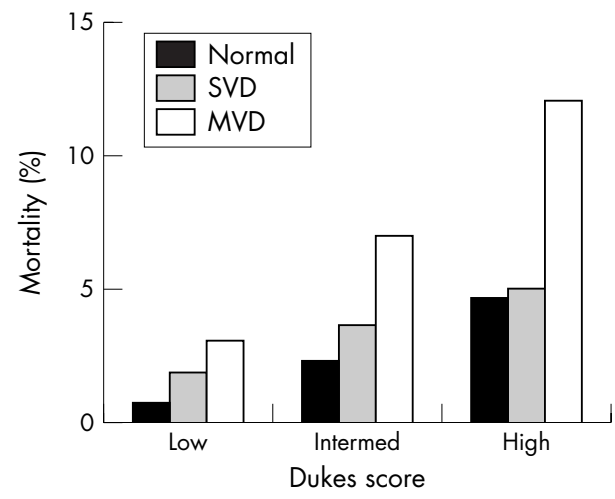

Figure 6 Combination of clinical risk assessment using Duke's score with extent of stress echocardiography abnormality, ( $n=5375$ patients). ${ }^{17}$
Duke's score, and resting LV function data. Furthermore, stress echocardiography was able to subclassify risk in all grades of Duke's score (fig 6). ${ }^{17}$ The greatest benefit of exercise echocardiography is in the evaluation of patients at intermediate risk by Duke treadmill score, which accounted for $40 \%$ of the patients in this study. ${ }^{17}$

In another study involving 1874 patients, McCully and colleagues $^{23}$ sought to define prognostic implications of the extent and severity of exercise echocardiography abnormalities in patients with good exercise capacity. They found that an increase in LV size after exercise identified patients who were likely to have had significantly more cardiac events compared to those whose LV size diminished after exercise. Similar predictive power of a positive test was demonstrated using dobutamine stress echocardiography in a study involving 3156 patients, where patients were followed up for nine years. ${ }^{24}$ Ischaemia and the extent of abnormal wall motion were independent predictors of cardiac death. Furthermore, the type of dobutamine response predicted outcome. Patients who showed resting LV dysfunction with additional ischaemia had the worst outcome compared to ischaemia alone, which pathophysiologically suggests greater amounts of myocardium in jeopardy (fig 7). In addition, global $\chi^{2}$ values improved incrementally over clinical and resting LV function data, when results of dobutamine stress data was added (fig 8). In another study involving 7333 patients undergoing pharmacological stress echocardiography, the outcome of an abnormal scan provided independent and incremental value over and above clinical data. ${ }^{18}$ The prognostic value of pharmacological stress echocardiography, relative to coronary angiography, was addressed in a subgroup of 4037 patients who underwent coronary angiography without an intervention. Coronary arteriographic data did not add significant predictive power to the model compared with stress echocardiographic variables (fig 9).

\section{Risk stratification in elderly, women, and diabetes mellitus patients}

The powerful impact of stress echocardiography in risk stratifying patients is seen in all subsets of patient populations irrespective of clinical markers of prognosis such as age, sex, and diabetes mellitus. In a study involving 5798 consecutive patients who underwent exercise echocardiography for evaluation of known or suspected CAD, no significant difference was noted in the ability of stress echocardiography to predict outcome between males and females, although significantly higher events occurred in males. ${ }^{25}$ In a study of 2632 patients over 65 years of age, stress echocardiography

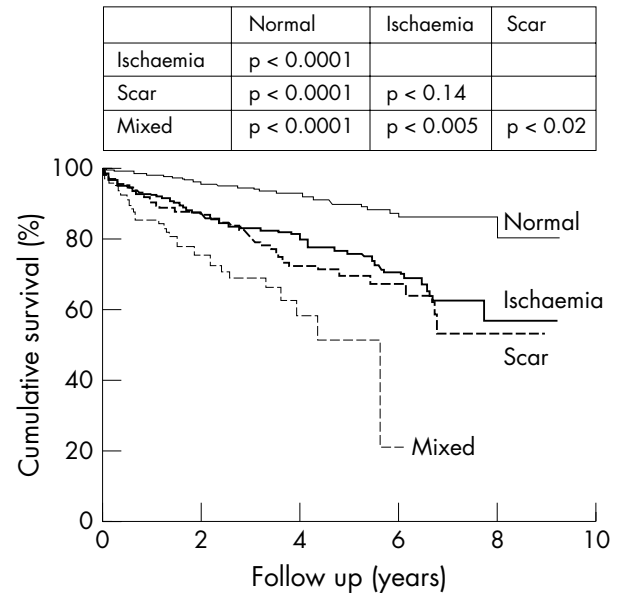

Figure 7 Cardiac mortality after dobutamine stress echocardiography ( $\mathrm{n}=3156$ patients)..$^{24}$ 

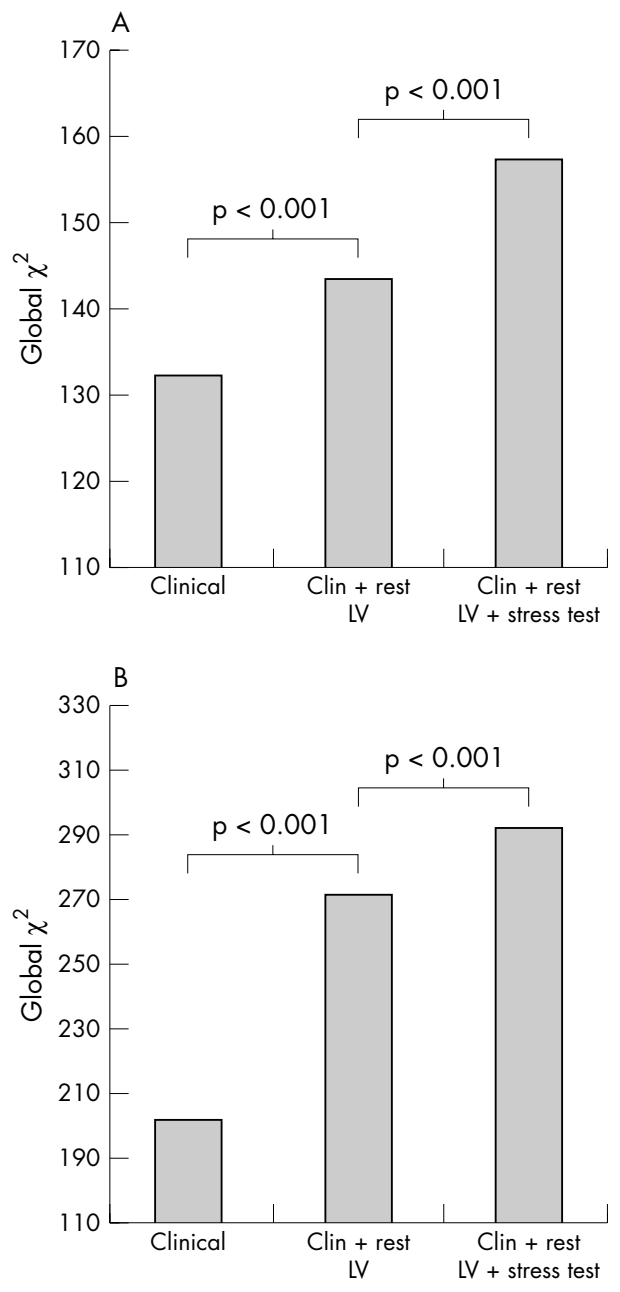

Figure 8 Incremental value of dobutamine stress echocardiography for predicting (A) total mortality and (B) cardiac mortality. ${ }^{24}$

provided incremental and independent information over and above clinical and exercise ECG data. ${ }^{26}$ In patients with diabetes mellitus it is important to identify the risk of cardiac events as these patients are at high risk of CAD. In a study of 563 patients, a normal stress echocardiogram predicted the absence of any cardiac event for up to two years with an annual cardiac event of $0.6 \%$ per year over a three year follow up. ${ }^{27}$ Furthermore, the degree of abnormality on an exercise echocardiogram correlated with increasing cardiac events (fig 10). The ability of stress echocardiography to assess both resting and stress LV function at one sitting makes it a powerful technique to assess both short and long term prognosis in all subsets of patients.

Risk stratification after acute myocardial infarction Thombolysis is widely used in the UK in patients with evolving ST elevation acute myocardial infarction. Despite its early use many patients are left with significant residual LV dysfunction. This residual LV dysfunction may be caused by post-ischaemic stunning or myocardial necrosis. Postischaemic stunning implies good prognosis as patients almost always recover LV function in the absence of residual flow limiting infarct related artery stenosis. Echocardiography during low doses of dobutamine demonstrates increased contractility in these dysfunctional segments. Several studies have confirmed the ability of dobutamine echocardiography to discriminate accurately between stunned and necrotic myocardium after acute myocardial infarction..$^{28}$ Five

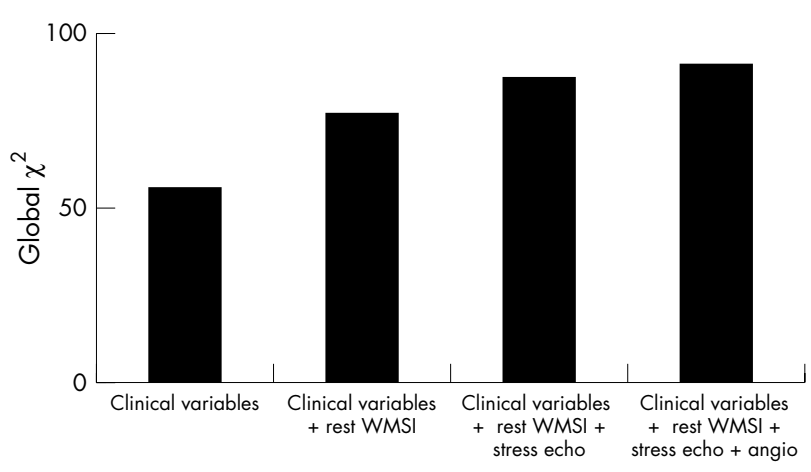

Figure 9 Bar graph showing the incremental value of pharmacologic stress echocardiography results compared to clinical characteristics and coronary angiography for the prediction of cardiac outcome in 4037 patients who underwent pharmacologic stress echocardiography and coronary angiography. WMSI, wall motion score index. ${ }^{19}$

studies involving 209 patients compared radionuclide perfusion imaging with dobutamine echocardiography in the same population for the prediction of recovery of function in dysfunctional segments after acute myocardial infarction. ${ }^{13}$ Dobutamine echocardiography demonstrated similar sensitivity but significantly higher specificity for the prediction of recovery of function. Lower specificity of SPECT is probably caused by a combination of technical and pathophysiological issues, such as lower resolution, shine through phenomenon, relatively increased extraction rates of tracers at lower myocardial blood flow, and the detection of islands of viable tissue in a predominantly necrotic myocardium. The lack of dobutamine induced contractile response indicating a poor prognosis is demonstrated by at least two recent studies. In one study, dobutamine echocardiography was performed in 214 patients at $12(6)$ days after acute myocardial infarction. ${ }^{30}$ During a follow up period of 9 (7) months, 12 cardiac deaths occurred. Absence of dobutamine response resulted in greater incidence of hard cardiac events. Another study, comprising 212 patients, performed in the UK in a similar group of patients performed 4 (3) days after acute myocardial infarction and with a longer follow up period, for the first time indicated that the most powerful marker of mortality is the lack of dobutamine response of the infarct related region, irrespective of whether patients subsequently underwent revascularistion or medical treatment. ${ }^{31}$

Another strategy for a comprehensive evaluation of a patient after uncomplicated acute myocardial infarction is to ascertain whether the infarct related viable myocardium is subtended by flow limiting coronary artery stenosis and/or whether the patient has remote ischaemia from the site of infarction. Exercise ECG is still widely used to address this question. Several studies have indicated that imaging techniques such as stress echocardiography or radionuclide perfusion imaging are superior to exercise ECG in defining subgroups of patients at low and high risk for future cardiac events. $^{32}{ }^{33}$ In a recent study Carlos and colleagues ${ }^{30}$ provided compelling evidence for the use of dobutamine echocardiography after acute myocardial infarction to accurately risk stratify patients. The authors studied 214 patients and performed dobutamine stress echocardiography within 2-7 days after acute myocardial infarction. Of these patients, $90 \%$ underwent coronary arteriography and 39\% underwent revascularisation, blinded to stress echocardiography data. Patients were followed up for a mean of 16 months for cardiac death, non-fatal acute myocardial infarction, unstable angina, ventricular tachycardia, and heart failure. When the results of stress echocardiography were compared with clinical and angiographic variables, the only independent 


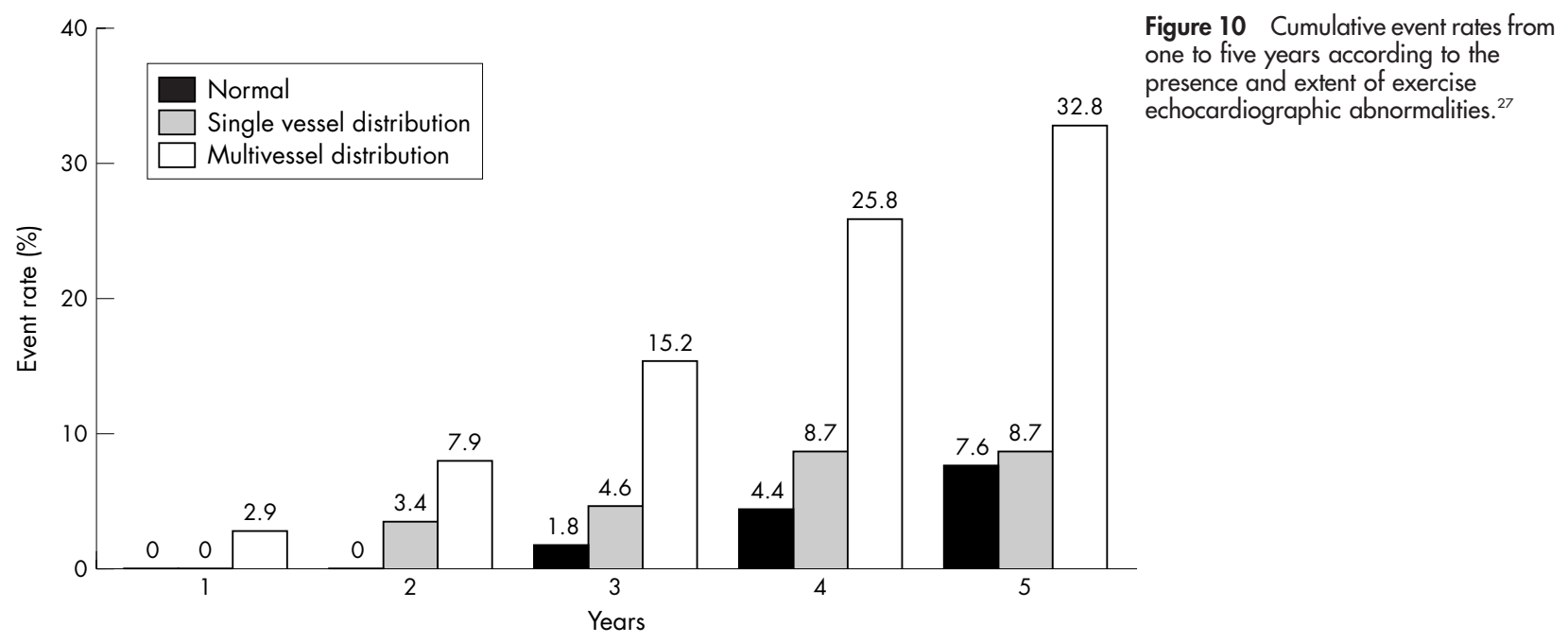

predictors of cardiac events were from stress echocardiographic variables. These variables were the extent of nonresponsive dobutamine segments (lack of myocardial viability) and presence of abnormal wall motion in multiple vascular territories. Indeed, in the revascularised group, extent of non-viable myocardium was the only predictor of cardiac events, while in the medically treated group both extent of non-viable myocardium and stress echocardiographic indicators of multi-vessel disease were independent predictors of cardiac events. Notably, the findings of dobutamine stress echocardiography were better at risk stratifying patients than the coronary angiography data (fig 11).

Thus, the above study shows that following acute myocardial infarction and thrombolysis, uncomplicated and stable patients may be first risk stratified using dobutamine stress echocardiography. Presence of myocardial viability and/or presence of stress echo demonstrated multi-vessel disease should triage these patients for coronary arteriography and revascularisation.

\section{Detection of hibernating myocardium}

Heart failure has significant mortality, morbidity, and cost complications. CAD accounts for $60 \%$ of patients with heart failure. A recent meta-analysis has shown the beneficial effect of revascularisation in heart failure only in patients with hibernating myocardium. ${ }^{34}$ Thus, considerable interest has been generated in using techniques that accurately identify hibernating myocardium.
Echocardiography can detect hibernating myocardium during dobutamine infusion through eliciting an enhanced contractile response by recruiting contractile proteins. Schultz and colleagues ${ }^{35}$ studied the impact of dobutamine infusion on regional mechanical and metabolic function in open chest anaesthetised pigs undergoing partial occlusion of the left anterior descending coronary artery. During occlusion, dobutamine infusion resulted in transient improvement of regional mechanical function. It is conceivable that contractile response may be elicited by dobutamine in a post-ischaemic stunned myocardium where the blood flow is normal at rest. However, it has also been suggested that where there is reduced myocardial blood flow at rest, it is likely that stimulation of the myocardium by dobutamine may precipitate myocardial ischaemia by increasing the oxygen demand beyond the already compromised threshold. ${ }^{35}$ In a study from Senior and colleagues ${ }^{36}$ involving 31 patients with a predominant history of heart failure and severe LV dysfunction ( $\mathrm{LV}$ ejection fraction $=25(9 \%)$ ), $39 \%$ of the severely dysfunctional segments showed normal perfusion, 32\% showed moderately reduced perfusion, and $29 \%$ were severely underperfused. Contractile response during dobutamine was similar in the normally perfused and moderately underperfused dysfunctional segments. Despite the majority of the segments being hypoperfused, the positive and negative predictive values of low dose dobutamine echocardiography for recovery of function of the dysfunctional segments following revascularisation were $88 \%$ and $85 \%$, respectively.

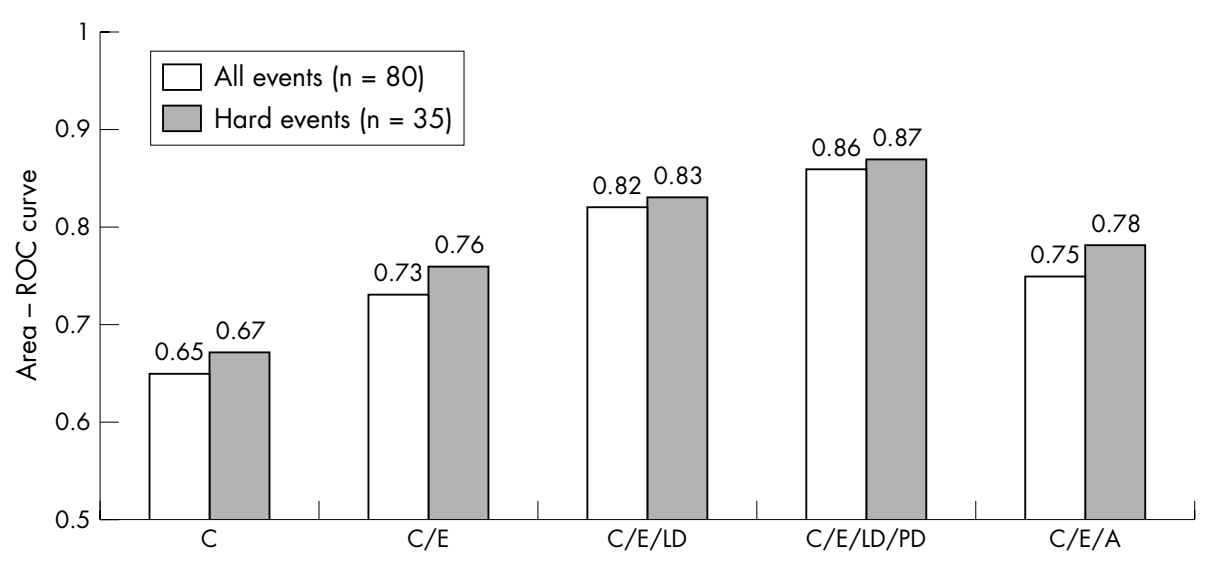

Figure 11 Incremental value of dobutamine stress echocardiography performed early after acute myocardial infarction. A, coronary arteriography; $C$, clinical; $E$, resting echocardiography; LD, low dose dobutamine; PD, peak dose dobutamine. ${ }^{30}$ 
Table 1 Accuracy of imaging techniques for detection of myocardial viability

\begin{tabular}{llll}
\hline & $\begin{array}{l}\text { No. of } \\
\text { patients }\end{array}$ & $\begin{array}{l}\text { Sensitivity } \\
(\%)\end{array}$ & $\begin{array}{l}\text { Specificity } \\
(\%)\end{array}$ \\
\hline${ }^{18}$ F-FDG PET & 332 & 88 & 73 \\
${ }^{201}$ Thallium re-injection & 209 & 86 & 47 \\
${ }^{201}$ Thallium rest- & 145 & 90 & 54 \\
$\begin{array}{l}\text { redistribution } \\
\text { 99mTC MIBI }\end{array}$ & 207 & 83 & 69 \\
$\begin{array}{l}\text { Dobutamine } \\
\text { echocardiography }\end{array}$ & 448 & 84 & 81 \\
\hline
\end{tabular}

${ }^{18} \mathrm{~F}$-FDG PET, ${ }^{18} \mathrm{~F}$-fluorodeoxyglucose positron emission tomography $99 \mathrm{mTC}$ MIBI, technetium-99m methoxyisobutylisonitrile.

The mechanism by which dobutamine stimulation elicits a contractile response in hypoperfused dysfunctional segments, without precipitating ischaemia, is through an increase in myocardial blood flow. ${ }^{37}$ The percentage increase in blood flow during dobutamine infusion in the dysfunctional myocardium approached that of normal myocardial regions; this was also confirmed by Krivokapich and colleagues. ${ }^{38}$ Another potential mechanism whereby the contractile response may be elicited during dobutamine, despite reduced resting myocardial flow, is through the peripheral vasodilator effect of dobutamine, which causes reduction in LV size and end systolic wall stress by reducing afterload, thus increasing systolic wall thickening for the same myocardial blood flow.

A summary of the diagnostic accuracy of different imaging techniques for the prediction of reversible dysfunction in patients with chronic ischaemic LV dysfunction is shown in table $1 .{ }^{39}$ The overall sensitivity and specificity of low dose dobutamine echocardiography for predicting recovery of regional function following revascularisation was $84 \%$ and $81 \%$, respectively, and was comparable to more expensive techniques for the detection of hibernating myocardium. ${ }^{39}$ The specificity of dobutamine echocardiography was better than SPECT for the reasons stated previously. Furthermore, studies have now shown that hibernating myocardium depicted by dobutamine echocardiography predicted reverse LV remodelling and improved survival when such patients underwent revascularisation. ${ }^{40-43}$ However, randomised trials are under way to address the relative benefit of revascularisation versus medical treatment in patients with hibernating myocardium. ${ }^{44}$

\section{Cost effectiveness of stress echocardiography}

One of the major arguments against the early performance of a stress imaging test as opposed to the widely used stress ECG with known or suspected CAD would be the greater cost implications of such a strategy. However, this argument does not take into account differences in downstream costs that may arise from the use of a less accurate initial test, which may precipitate the inappropriate use of more expensive investigations, as well as failing to identify patients who go on to potentially costly complications. In contrast to radionuclide imaging, stress echocardiography represents a smaller initial cost increment from the stress ECG alone. One major study

Table 2 Risk stratification by exercise echocardiography and exercise ECG ${ }^{45}$

\begin{tabular}{llll}
\hline & Low risk & Intermediate risk & High risk \\
\hline Exercise & & & \\
echocardiography & $51 \%$ & $27 \%$ & $22 \%$ \\
Exercise ECG & $24 \%$ & $51 \%$ & $25 \%$ \\
p Value & $\mathrm{p}<0.001$ & $\mathrm{p}<0.001$ & $\mathrm{p}=\mathrm{NS}$ \\
\hline
\end{tabular}

supported by the American Society of Echocardiography addressed the cost effectiveness of stress echocardiography compared to stress ECG for patients with known or suspected $\mathrm{CAD}^{45}$ Marwick and colleagues studied 7656 patients undergoing exercise testing of whom half underwent stress echocardiography. Cox proportional hazards models, risk adjusted for pre-test likelihood of $\mathrm{CAD}$, were used to estimate time to cardiac death or acute myocardial infarction. Costs, including diagnostic and revascularisation procedures, hospitalisation, and events, were calculated. Compared to exercise ECG, stress echocardiography identified more patients as low risk and fewer at intermediate and high risk (table 2). Survival was greater in low and intermediate risk and less in high risk patients and was better classified by stress echocardiography than exercise ECG.

Although initial procedural costs were greater, exercise echocardiography was associated with a greater incremental life expectation (0-2 years), and a lower cost of additional diagnostic procedures when compared with exercise ECG. Exercise echocardiography was more cost effective (€2615 per life year saved) than exercise ECG (table 3). The authors further concluded that patients with symptoms who need non-invasive evaluation are less likely to undergo coronary angiography, and hence revascularisation, if stress echocardiography is performed in preference to exercise ECG.

In another recent randomised study conducted in the UK, 302 patients presenting to the emergency department with suspected CAD, but with non-diagnostic ECG and negative cardiac enzymes, underwent stress echocardiography or exercise ECG for further risk stratification. Stress echocardiography was superior to exercise ECG in risk stratifying these patients to low and high risk groups (fig 12) and resulted in a higher rate of early hospital discharge in the stress echocardiography arm. Significantly more patients underwent additional testing based on exercise ECG results compared to stress echocardiography. The study showed that the stress echocardiography strategy resulted in potentially significant cost saving compared with stress ECG. ${ }^{46}$

\section{Limitations of stress echocardiography}

The often quoted limitations of stress echocardiography are suboptimal image quality (10-15\%) and lack of quantitation. ${ }^{47}$ Both limitations make stress echocardiography more operator dependent and less reproducible. In a study by Nagel and colleagues, ${ }^{47}$ dobutamine stress echocardiography was compared to high resolution stress magnetic resonance imaging. ${ }^{47}$ Magnetic resonance imaging was found to be superior to echocardiography for the diagnosis of CAD. In this study nearly $50 \%$ of stress echocardiography images were of modest or of non-diagnostic quality. With the advent of harmonic and contrast imaging, both now widely used, suboptimal echocardiographic images-for example, in cases of obesity and lung disease-are becoming rare. It has been shown convincingly in large multi-centre trials that the

\begin{tabular}{|c|c|c|}
\hline & $\begin{array}{l}\text { Exercise } \\
\text { echocardiography } \\
\text { ( } n=3860 \text { ) }\end{array}$ & $\begin{array}{l}\text { Exercise ECG } \\
(n=3796)\end{array}$ \\
\hline Years of life saved & 89459 & 88464 \\
\hline Average life expectancy (years) & $\begin{array}{l}23.54 \\
€ 17419657\end{array}$ & $\begin{array}{l}23.52 \\
€ 16842611\end{array}$ \\
\hline $\begin{array}{l}\text { Total observed cost } \\
\text { Estimated lifetime cost }\end{array}$ & $\begin{array}{l}€ 17419657 \\
€ 373477439\end{array}$ & $€ 370874298$ \\
\hline Total cost difference & $€ 2603141$ & \\
\hline $\begin{array}{l}\text { Approximate cost per life } \\
\text { year saved }\end{array}$ & $€ 2615$ & \\
\hline
\end{tabular}




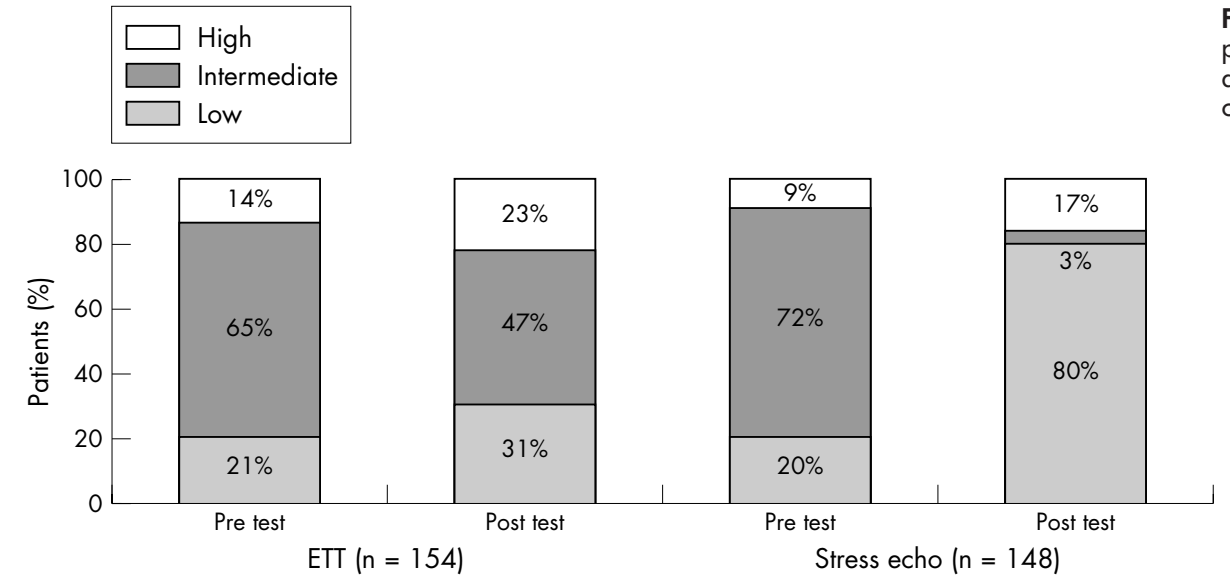

Figure 12 Risk stratification pre- and post-tests in 302 patients presenting to accident and emergency with acute chest pain. ${ }^{46}$

newer modes of imaging have improved not only image quality but provided reproducible image interpretation. ${ }^{48} 49$ Training issues which include correct acquisition of images, recognising artefacts, performance, and interpretation of studies under a trained cardiologist are of paramount importance in stress echocardiography. For example, interpreting isolated basal inferior and septal wall abnormalities often leads to increased false positive scans.

\section{Future of stress echocardiography}

Rapid development of myocardial perfusion imaging using ultrasound contrast agents now may allow simultaneous assessment of both function and perfusion (fig 13). ${ }^{50-52}$ This may allow not only improved assessment of wall motion, both at rest and during stress, but may also enhance the diagnostic value of stress echocardiography for the detection of CAD. ${ }^{53}$ Myocardial perfusion can be quantified which would further enhance the diagnostic ability of stress echocardiography. ${ }^{54}$ With refinements in tissue Doppler imaging, assessment of myocardial velocity allows quantitative and reproducible assessment of myocardial wall motion. In a recent multi-centre study, assessment of myocardial velocity provided parameters for quantitative evaluation of wall motion for the diagnosis of CAD. ${ }^{5}$ Thus, future usage of ultrasound contrast agents and tissue Doppler imaging is likely to form an integral part of stress echocardiography.
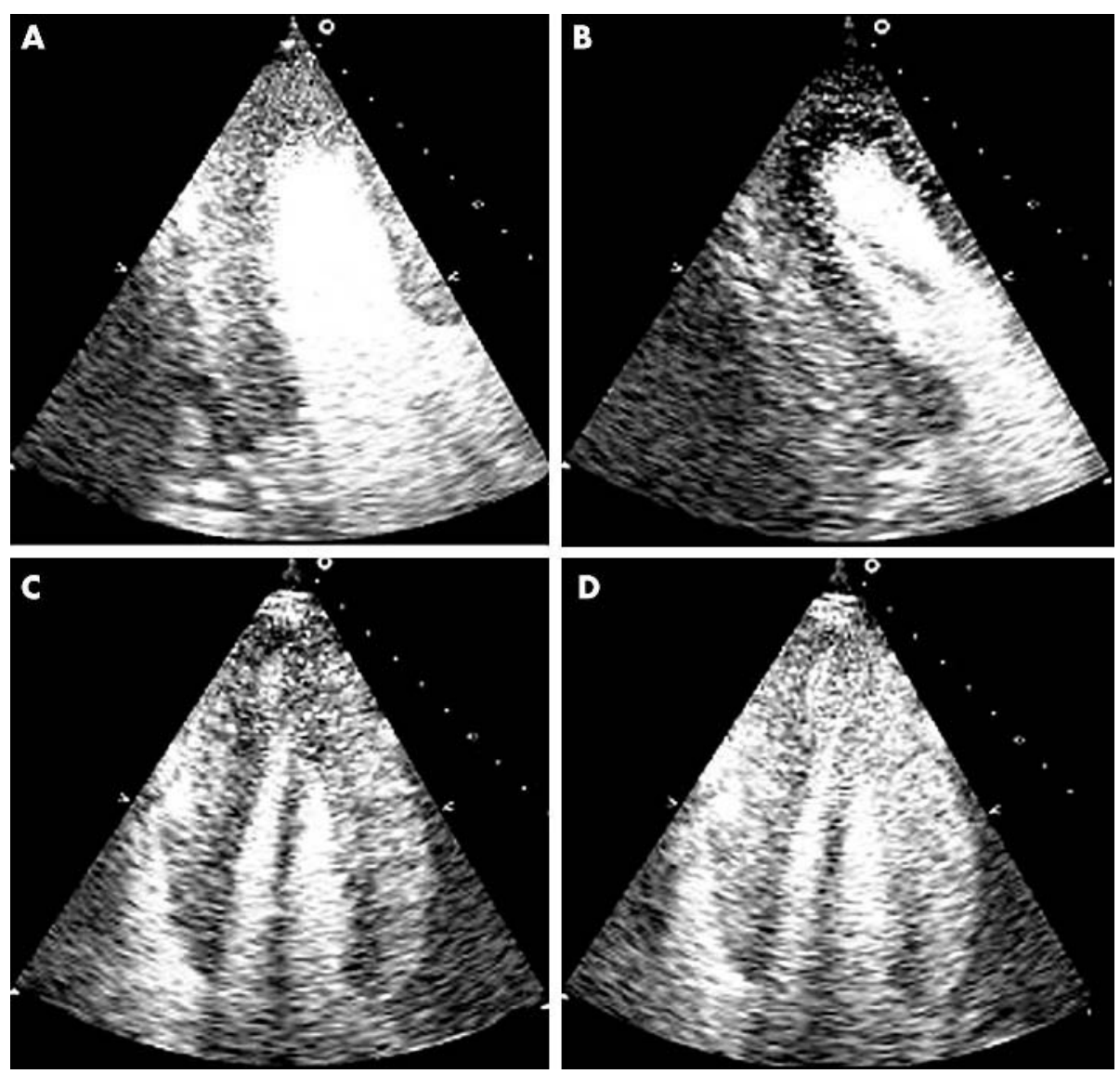

Figure 13 Contrast destructionreperfusion study during dobutamine stress echocardiography in a patient with $70 \%$ mid left anterior descending coronary artery lesion and a severely diseased right coronary artery with a $90 \%$ lesion. Panels $A$ and $B$ are end systolic apical two chamber views at rest (A) three beats post-destruction and at peak stress (B) one beat postdestruction. A significant subendocardial defect is seen in the anterior, apical, and inferior walls. Panels $C$ and $D$ are both apical four chamber views at peak stress. Panel $C$ is one beat post-destruction and shows an apical and septal sub-endocardial defect. It also demonstrates aneurysmal pouching of the apex which was even more evident on real time images. Panel $D$ is three beats post-destruction and the sub-endocardial defects have filled in although the apical shape distortion is still evident (post-ischaemic stunning). 


\section{CONCLUSIONS}

Clinical evidence involving more than 30000 patients in multiple controlled trials has established stress echocardiography as a powerful non-invasive tool, not only for the diagnosis of CAD, but also for risk stratifying patients with known or suspected CAD. Recently in the UK the National Institute for Clinical Excellence (NICE) has evaluated SPECT, comparing it with exercise ECG testing, and has recommended its use ${ }^{56}$; however, the original remit did not include assessment of stress echocardiography techniques. As outlined in this review there is overwhelming evidence that stress echocardiography is equivalent to SPECT in the situations outlined by NICE; it is also cheaper, easier to set up, and does not involve radioactivity. Furthermore, recent developments in the field of echocardiography contrast facilitate simultaneous assessment of myocardial function and perfusion.

\section{Authors' affiliations}

R Senior, Northwick Park Hospital, Harrow, UK

M Monaghan, King's College Hospital, London, UK

H Becher, John Raddliffe Hospital/Cardiac Clinical Centre, Oxford, UK

J Mayet, St Mary's Hospital, Paddington, London, UK

P Nihoyannopoulos, Imperial College Faculty of Medicine, National

Heart \& Lung Institute, Hammersmith Hospital, London, UK

\section{REFERENCES}

1 Detrano R, Gianrossi R, Froelicher V. The diagnostic accuracy of the exercise electrocardiogram: a meta-analysis of 22 years of research. Prog Cardiovasc Dis 1989;32:173-206.

2 Marwick TH. Current status of non-invasive techniques for the diagnosis of myocardial ischemia. Acta Clin Belg 1992;47:1-5.

3 Pryor DB, Harrel FEJ, Lee KL, et al. Estimating the likelihood of significant coronary artery disease. Am J Med 1983;75:771-80.

4 Hoffmann R, Marwick TH, Poldermans D, et al. Refinements in stress echocardiographic techniques improve inter-institutional agreement in interpretation of dobutamine stress echocardiograms. Eur Heart $J$ 2002;23:821-9.

5 Mälder CF, Payne N, Wilkenshoff U, et al. Non-invasive diagnosis of coronary artery disease by quantitative stress echocardiography. Eur Heart $J$ 2003;24:1584-94.

6 Rainbird AJ, Mulvagh SL, McCully OJK, et al. Contrast dobutamine stress echocardiography: clinical practice assessment in 300 consecutive patients. J Am Soc Echocardiogr 2001; 14:378-85

7 Tsoukas A, Ikonomidis I, Cokkinos P, et al. Significance of persistent left ventricular dysfunction during recovery after dobutamine stress echocardiography. J Am Coll Cardiol 1997;30:621-6.

8 Senior R, Lahiri A. Enhanced detection of myocardial ischemia by stress dobutamine echocardiography utilizing the "biphasic" response of wall thickening during low and high dose dobutamine infusion. J Am Coll Cardiol 1995;26:26-32

9 Soman P, Lahiri A, Senior R. Vasodilator stress induces infrequent wall thickening abnormalities compared to perfusion defects in mild to moderate coronary artery disease: implications for the choice of imaging modality with vasodilator stress. Echocardiography 2004;21:307-12.

10 Beleslin BD, Osojic M, Diordjevic-Dikie A, et al. Integrated evaluation of relation between coronary lesion features and stress echocardiography results: the importance of coronary lesion morphology. J Am Coll Cardiol 1999;33:717-26.

11 Marwick TH. Stress echocardiography. Heart 2003;89:113-8.

12 Fleischmann KE, Hunink MGM, Kuntz KM, et al. Exercise echocardiography or exercise SPECT imaging? JAMA 1998;280:913-20.

13 Geleijnse ML, Elhendy A. Can stress echocardiography compete with perfusion scintigraphy in the detection of coronary artery disease and cardiac risk assessment? Eur J Echocardiogr 2000;1:12-21.

14 Roger VL, Pellikka PA, Oh JK, et al. Identification of multivessel coronary artery disease by exercise echocardiography. J Am Coll Cardiol 1994;24:109-14.

15 Senior R, Khattar R, Lahiri A. Value of dobutamine stress echocardiography for the detection of multivessel coronary artery disease. Am J Cardiol 1998;81:298-301.

16 Smart SC, Knickelbine T, Malik F, et al. Dobutamine-atropine stress echocardiography for the detection of coronary artery disease in patients with left ventricular hypertrophy. Importance of chamber size and systolic wall stress. Circulation 2000;101:258-63.

17 Marwick TH, Case C, Vasey C, et al. Prediction of mortality by exercise echocardiography. A strategy for combination with the Duke treadmill score. Circulation 2001;29:2566-71.

18 McCully RB, Roger VL, Mahoney DW, et al. Outcome after normal exercise echocardiography and predictors of subsequent cardiac events: follow-up of 1,325 patients. J Am Coll Cardiol 1998;31:144-9.
19 Sicari R, Pasanisi E, Venneri L, et al. Stress echo results predict mortality: a large-scale multicenter prospective international study. J Am Coll Cardiol 2003;41:589-95

20 Chung G, Krishnamani R, Senior R. Prognostic value of normal stress echocardiogram in patients with suspected coronary artery disease - A British general hospital experience. Int J Cardiol 2004;94:181-6.

21 Geleijnse ML, Elhendy A, Van Domburg RT, et al. Cardiac imaging for risk stratification with dobutamine atropine stress testing in patients with chest pain. Circulation 1997;96:137-47.

22 Olmos LI, Dakik H, Cordon R, et al. Long-term prognostic value of exercise echocardiography compared with exercise 201TL, ECG, and clinical variables in patients evaluated for coronary artery disease. Circulation 1998;98:2679-86.

23 McCully RB, Roger VL, Mahoney DW. Outcome after abnormal exercise echocardiography for patients with good exercise capacity. J Am Coll Cardiol 2002;39:1778-83.

24 Marwick TH, Case C, Sawada S, et al. Prediction of mortality using dobutamine echocardiography. J Am Coll Cardiol 2001;37:754-60.

25 Arruda-Olson AM, Juracan EM, Mahoney DW, et al. Prognostic value of echocardiography in 5,798 patients: is there a gender difference? J Am Coll Cardiol 2002;39:625-31.

26 Arruda AM, Das MK, Roger VL, et al. Prognostic value of exercise echocardiography in 2,632 patients $\geqslant 65$ years of age. J Am Coll Cardiol 2001;37:1345-52.

27 Elhendy A, Arruda AM, Mahoney, et al. Prognostic stratification of diabetic patients by exercise echocardiography. J Am Coll Cardiol 2001;37:1551-7.

28 Pierard L, De Landsheere CM, Berthe C, et al. Identification of viable myocardium by echocardiography during dobutamine infusion in patients with myocardial infarction after thrombolytic therapy: comparison with positron emission tomography. J Am Coll Cardiol 1990;15:1021-31.

29 Barilla F, Gheorghiade M, Alam M, et al. Low dose dobutamine in patients with acute myocardial infarction identifies viable but not contractile myocardium and predicts the magnitude of improvement in wall motion abnormalities in response to coronary revascularisation. Am Heart J 1991;122:1522-31.

30 Carlos ME, Smart SC, Wynsen JC, et al. Dobutamine stress echocardiography for risk stratification after myocardial infarction. Circulation 1997;95:1402-10.

31 Swinburn J, Senior R. Extent of non-viability, not ischaemia, predicts mortality after myocardial infarction. Heart 2004;90(suppl II):A35.

32 Ryan T, Armstrong WF, O'Donnell JA, et al. Risk stratification after acute myocardial infarction by means of exercise two-dimensional echocardiography. Am Heart J 1987;1 14:1305-16.

33 Gibson RS, Watson DD, Carddock GB, et al. Prediction of cardiac events after uncomplicated myocardial infarction: a prospective study comparing predischarge exercise thallium-201 scintigraphy and coronary angiography. Circulation 1983:68:321-36.

34 Allman KC, Shaw $\amalg$, Hachamovitch R, et al. Myocardial viability testing and impact of revascularization on prognosis in patients with coronary artery disease and left ventricular dysfunction: a meta-analysis. J Am Coll Cardiol 2002;39:1151-8.

35 Schulz R, Guth BD, Peiper K, et al. Recruitment of an inotropic reserve in moderately ischaemic myocardium at the expense of metabolic recovery. A model of short term hibernation. Circ Res 1992;70:1282-95.

36 Senior R, Lahiri A. Dobutamine echocardiography predicts functional outcome after revascularisation in patients with dysfunctional myocardium irrespective of the perfusion pattern on resting thallium-201 imaging. Heart 1999;82:668-73.

37 Sun KT, Czernin J, Krivokapich J, et al. Effects of dobutamine stimulation on myocardial blood flow, glucose metabolism, and wall motion in normal and dysfunctional myocardium. Circulation 1996;94:3146-54.

38 Krivokapich J, Czernin J, Schelbert HR. Dobutamine positron emission tomography. Absolute quantification of rest and dobutamine myocardial blood flow and correlation with cardiac work and percent diameter stenosis in patients with and without coronary artery disease. J Am Coll Cardiol 1996;28:565-72

39 Bax JJ, Wiins W, Cornel JH, et al. Accuracy of currently available techniques for prediction of functional recovery after revascularisation in patients with left ventricular dysfunction due to chronic disease: Comparison of pooled data. J Am Coll Cardiol 1997;30:1451-60.

40 Senior R, Kaul S, Lahiri A. Myocardial viability on echocardiography predicts long term survival after revascularisation in patients with ischaemic congestive heart failure. J Am Coll Cardiol 1999;33:1848-54.

41 Afridi I, Grayburn PA, Panza JA, et al. Myocardial viability during dobutamine echocardiography predicts survival in patients with coronary artery disease and severe left ventricular dysfunction. J Am Coll Cardiol 1998;32:921-6.

42 Senior R, Lahiri A, Kaul S. Effect of revascularisation on left ventricular remodelling in patients with heart failure from severe chronic ischaemic left ventricular dysfunction. Am J Cardiol $2001 ; 88: 624-9$.

43 Meluzin J, Cerny J, Frelich M, et al. Prognostic value of the amount of dysfunctional but viable myocardium in revascularisatized patients with coronary artery disease and left ventricular dysfunction. J Am Coll Cardiol 1998;32:912-20

44 Cleland JG, Freemantle N, Ball SG, et al. The heart failure revascularisation trial (HEART): rationale, design and methodology. Eur J Heart Fail 2003;5:295-303.

45 Marwick TH, Shaw L, Case C, et al. Clinical and economic impact of exercise electrocardiography and exercise echocardiography in clinical practice. Eur Heart J 2003;24:1153-63. 
46 Jeetley P, Burden L, Senior R. Superior risk stratification by stress echocardiography compared with exercise electrocardiography in troponin negative acute chest pain - A prospective randomised trial. Heart 2004;90(suppl II):A33

47 Nagel E, Lehmkuhl HB, Bocksch W, et al. Noninvasive diagnosis of ischemiainduced wall motion abnormalities with the use of high-dose dobutamine stress MRI: comparison with dobutamine stress echocardiography. Circulation 1999;99:763-70.

48 Anand DV, Theodosiadis ID, Senior R. Improved interpretation of dobutamine stress echocardiography following 4 months of systematic training inpatients following acute myocardial infarction. Eur J Echocardiogr 2004;5:12-7.

49 Nanda NC, Kitzman DW, Dittrich HC, et al. Imagent improves endocardial border delineation, inter-reader agreement, and the accuracy of segmental wall motion assessment. Echocardiography 2003;20:151-61.

50 Porter TR, Xie F, Silver $M$, et al. Real-time perfusion imaging with low mechanical index pulse inversion Doppler imaging. J Am Coll Cardiol 2001;37:748-753.

51 Shimoni S, Zoghbi WA, Xie F. Real-time assessment of myocardial perfusion and wall motion during bicycle and treadmill exercise echocardiography: comparison with single photon emission computed tomography. J Am Coll Cardiol $2001 ; 37: 741-7$
52 Senior R, Lepper W, Pasquet A, et al. Myocardial perfusion assessment in patients with medium probability of coronary artery disease and no prior myocardial infarction: comparison of myocardial contrast echocardiography with ${ }^{99 \mathrm{~m} T c-S P E C T}$. Am Heart J 2004;147:1100-5

53 Moir S, Haluska BA, Jenkins C, et al. Myocardial contrast stress echocardiography for the assessment of coronary artery disease: incremental benefit of qualitative and quantitative approaches. Circulation 2004; 110:1108-13.

54 Peltier M, Vancraeynest D, Pasquet A, Assessment of the physiologic significance of coronary disease with dipyridamole real-time myocardial contrast echocardiography. Comparison with technetium- $99 \mathrm{~m}$ sestambi single-photon emission computed tomography and quantitative coronary angiography. J Am Coll Cardiol 2004:43:257-64.

55 Janardhanan R, Senior R. Accuracy of dipyridamole myocardial contrast echocardiography for the detection of residual stenosis of the infract-related artery and multivessel disease early after acute myocardial infarction. J Am Coll Cardiol 2004; 16:2247-52.

56 UK National Institute for Clinical Excellence, guidance on the role of MPI in the diagnosis and management of patients with angina and myocardial infarction (www.nice.org.uk/TA073 guidance).

\section{IMAGES IN CARDIOLOGY}

Patency of the right coronary artery following implantation of metallic stent demonstrated by multislice computed tomography

A 67 year old man presented to our hospital with chest pressure. A conventional angiogram revealed significant stenosis in the proximal portion of the right coronary artery (RCA) and percutaneous transluminal coronary angioplasty was therefore performed with successful stent implantation at the stenotic site. After three months, to evaluate patency of the metallic stent, ECG gated enhanced multislice computed tomography (CT) (Light Speed Ultra 16, General Electric, Milwaukee, Wisconsin, USA) was performed with a $0.625 \mathrm{~mm}$ slice thickness, helical pitch 3.25. Thirty seconds after intravenous injection of $100 \mathrm{ml}$ of iodinated contrast material $(350 \mathrm{mg} \mathrm{I} / \mathrm{ml}$ ), CT scanning was performed with retrospective ECG gated reconstruction and volume data were transferred to a workstation (Virtual Place Office, Azemoto, Tokyo, Japan).

Volume rendered images revealed the implanted stent graft in the proximal portion of the RCA (panel A). Axial source (panel B) and multiplanar reconstruction images of the long axis of the proximal portion of the RCA (panel C) clearly showed the patent lumen of the proximal portion of the RCA, surrounded by the implanted stent graft. Conventional coronary angiogram from the left anterior oblique direction revealed the patent lumen of the proximal portion of the RCA (arrowhead,
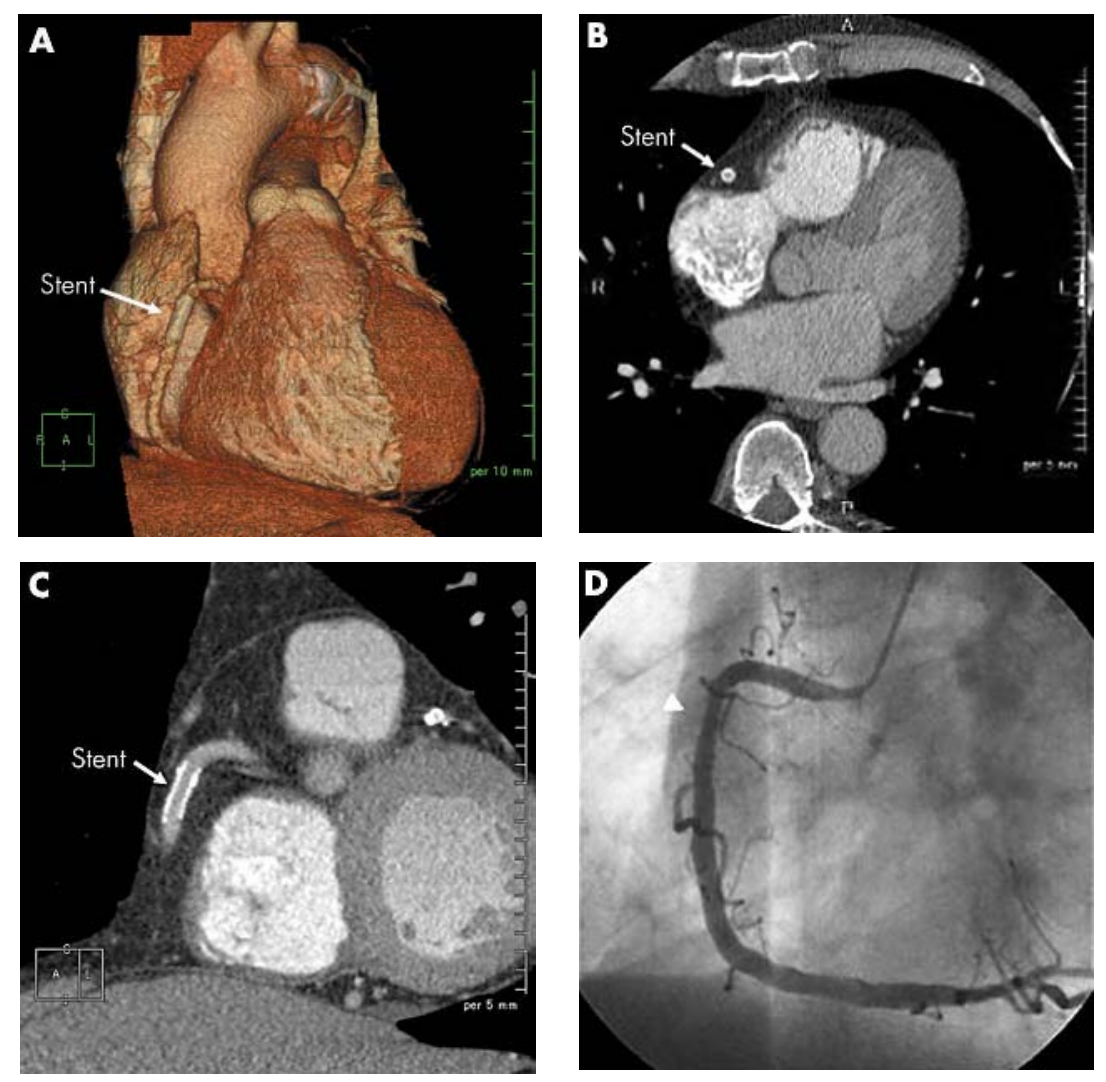

panel D), confirming the findings of the CT images shown in panel $\mathrm{C}$.
N Funabashi

N Komiyama I Komuro nobusada@ma.kcom.ne.jp 